\title{
Counting $k$-Marked Durfee Symbols
}

\author{
Kağan Kurşungöz \\ Department of Mathematics \\ The Pennsylvania State University, University Park, PA 16802 \\ kursun@math.psu.edu
}

Submitted: May 7, 2010; Accepted: Feb 5, 2011; Published: Feb 14, 2011

Mathematics Subject Classification: 05A15, 05A05

\begin{abstract}
An alternative characterization of $k$-marked Durfee symbols defined by Andrews is given. Some identities involving generating functions of $k$-marked Durfee symbols are proven combinatorially by considering the symbols not individually, but in equivalence classes. Also, a related binomial coefficient identity is obtained in the course.
\end{abstract}

A partition $\lambda$ of a positive integer $n$ is a nonincreasing sequence of positive integers $\lambda_{1} \geq \cdots \geq \lambda_{k}>0$ such that $n=\lambda_{1}+\cdots+\lambda_{k}[1$, Ch. 1$]$.

A pictorial representation for a partition is its Ferrers graph, a left indented table of dots such that the first row has $\lambda_{1}$ dots and so on. For instance, $36=9+7+7+5+4+2+2$ has the following Ferrers graph.

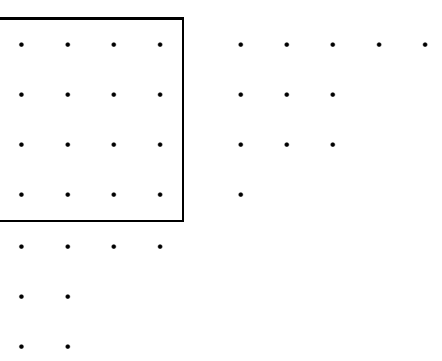

The largest square that can be fit in the upper left corner of a Ferrers graph is called the Durfee square. So the partition above has a Durfee square of side length 4 .

The rank of a partition is defined as the largest part minus the number of parts in a partition [5]. The rank of the above partition is +2 .

The conjugate $\lambda^{\prime}$ of a partition $\lambda$ is obtained by reflecting the Ferrers graph across its main diagonal. For the partition above, the conjugate is $7+7+5+5+4+3+3+1+1$ 
and has the following Ferrers graph.

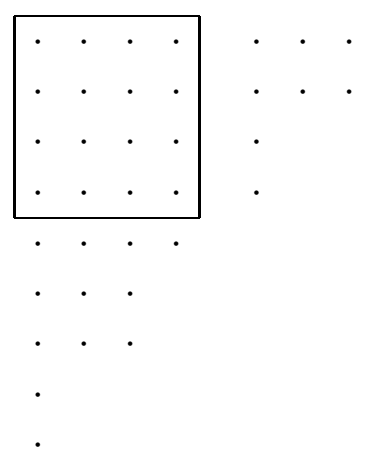

Using the Ferrers graph of a partition, we can form another representation of the same partition, the Durfee symbol [3]. It is a two-row array, with a subscript indicating the side length of the Durfee square. The top row is obtained by reading the conjugate partition of the smaller partition to the right of the Durfee square (recording the columns instead of rows), and the bottom row by reading the smaller partition below the Durfee square. Thus, the partition $36=9+7+7+5+4+2+2$ has Durfee symbol

$$
\left(\begin{array}{lllll}
4 & 3 & 3 & 1 & 1 \\
4 & 2 & 2 & &
\end{array}\right)_{4}
$$

Using the Durfee symbol, it is simple to find the rank; as it is the excess of the number of parts in the top row over the number of parts in the bottom row. Notice also that in terms of the Durfee symbol, finding the conjugate corresponds to interchanging the top and bottom row.

In [3], Andrews generalized this notion and defined $k$-marked Durfee symbols. He provided and proved a number of identities and congruences. He mainly used analytical methods, and left the combinatorial explanations to his results as open problems. In $\S 1$, an alternative definition of $k$-marked Durfee symbols is given. Using this alternative characterization, some combinatorial open problems listed at the end of [3] are solved. The method employed is to define equivalence classes of Durfee symbols of a given number, and to consider the possible ways to make the symbols in an equivalence class into $k$-marked Durfee symbols.

Part of the results presented in $\S 3$ appears in [4], where the authors use the same alternative characterization of $k$-marked Durfee symbols, but more direct combinatorial methods. In particular, they present a bijection, and a sieve to establish the symmetry, and the relation to ordinary Durfee symbols of the $k$-marked ones.

Also, Ji [8] proved the results in $\S 3$ along with many more open problems posed by Andrews in [3]. Ji's approach is essentially different from $\S 1$ and [4]. She defines the strict shifted $k$-marked Durfee symbols, shows how to obtain the original $k$-marked Durfee symbols from these, and then compares coefficients of certain terms on either sides of identities. 


\section{Definitions}

Definition 1.1. Let $\mathcal{S}_{d}\left(m_{1}, \ldots, m_{d}\right)$ denote the collection of Durfee Symbols with Durfee square of side length $d$ where the total number of appearances of $j$ in the listed Durfee symbols is exactly $m_{j}$ for $j=1, \ldots, d$. This is a subset of partitions of $n=d^{2}+\sum_{j=1}^{d} j m_{j}$,

$$
\begin{aligned}
& \text { Example: } \mathcal{S}_{2}(2,3)=\left\{\left(\begin{array}{ll}
222 & 11 \\
&
\end{array}\right)_{2},\left(\begin{array}{ll}
222 & 1 \\
& 1
\end{array}\right)_{2},\left(\begin{array}{ll}
222 & \\
& 11
\end{array}\right)_{2},\left(\begin{array}{ll}
22 & 11 \\
2 &
\end{array}\right)_{2},\right. \\
& \left(\begin{array}{ll}
22 & 1 \\
2 & 1
\end{array}\right)_{2},\left(\begin{array}{ll}
22 & \\
2 & 11
\end{array}\right)_{2},\left(\begin{array}{ll}
2 & 11 \\
22 &
\end{array}\right)_{2},\left(\begin{array}{ll}
2 & 1 \\
22 & 1
\end{array}\right)_{2},\left(\begin{array}{ll}
2 & \\
22 & 11
\end{array}\right)_{2},\left(\begin{array}{ll}
222 & 11
\end{array}\right)_{2}, \\
& \left.\left(\begin{array}{rl}
222 & 1
\end{array}\right)_{2},\left(\begin{array}{ll}
222 & 11
\end{array}\right)_{2}\right\} \text {. }
\end{aligned}
$$

Andrews extended the definition of Durfee symbols to odd Durfee symbols. In an odd Durfee symbol only odd numbers occur, and the Durfee square is reinterpreted. The detailed description can be found in [3].

Definition 1.2. Let $\mathcal{S}_{d}^{o}\left(m_{1}, \ldots, m_{d}\right)$ denote the collection of odd Durfee Symbols with Durfee square side of side length $d$ where the total number of appearances of $2 j-1$ in the listed odd Durfee symbols is exactly $m_{j}$ for $j=1, \ldots, d$. This is a subset of partitions of $n=\left(2 d^{2}-2 d+1\right)+\sum_{j=1}^{d}(2 j-1) m_{j}$.

We recall one more definition from [3, §4], but rewrite it in an alternative form. This alternative definition provides the base in [4] for the combinatorial explorations of some results stated in [3].

Definition 1.3. A $k$-Marked Durfee Symbol $\tau$ is a concatenation of $k$ two-row arrays $\left(\begin{array}{ccc}a_{i, m_{i}} & \cdots & a_{i, 1} \\ b_{i, n_{i}} & \cdots & b_{i, 1}\end{array}\right), i=1, \ldots, k$, with $k-1$ posts $p_{1}, \ldots, p_{k-1}$ in between. The first index indicates the mark. Either or both rows may be empty in an array, and the following monotonicity conditions hold:

$$
\begin{gathered}
a_{i, j} \leq a_{i, j+1}, \quad i=1, \ldots, k, \quad j=1, \ldots, m_{i}-1 \quad \forall \text { fixed } i, \\
b_{i, j} \leq b_{i, j+1}, \quad i=1, \ldots, k, \quad j=1, \ldots, n_{i}-1 \quad \forall \text { fixed } i, \\
\max \left\{a_{i, m_{i}}, b_{i, n_{i}}, p_{i-1}\right\} \leq p_{i} \leq \min \left\{a_{i+1,1}, b_{i+1,1}, p_{i+1}\right\} \quad i=1, \ldots, k
\end{gathered}
$$

where $p_{0}=1, p_{k}=d$, $d$ being the side length of the Durfee Square.

$$
\begin{aligned}
& \tau=\left(\begin{array}{cccccccc}
a_{k, m_{k}} & \cdots & a_{k, 1} & p_{k-1} & a_{k-1, m_{k-1}} & \cdots & a_{k-1,1} & p_{k-2} \\
b_{k, n_{k}} & \cdots & b_{k, 1} & & b_{k-1, n_{k-1}} & \cdots & b_{k-1,1} &
\end{array}\right. \\
& \left.\begin{array}{lllll}
\cdots & p_{1} & a_{1, m_{1}} & \cdots & a_{1,1} \\
\cdots & & b_{1, n_{1}} & \cdots & b_{1,1}
\end{array}\right)_{d}
\end{aligned}
$$

If we reinterpret the Durfee square $\left(2 d^{2}-2 d+1\right.$ instead of $\left.d^{2}\right)$, and allow odd numbers only, we get the definition of $k$-marked odd Durfee symbols. 
Definition 1.4. The excess of entries in the top row of ith array over the entries in the bottom row in the same array is the $i$ th rank of a $k$-marked Durfee symbol (or a $k$-marked odd Durfee symbol) $\tau$, denoted $r_{i}(\tau)$.

Notice that ordinary Durfee symbols can be regarded as 1-marked Durfee symbols, we will write $r(\tau)=r_{1}(\tau)$ in that case, since there is only one rank.

\section{Example:}

$$
\tau=\left(\begin{array}{lllll} 
& 4 & & 2 & 2 \\
6 & 5 & 3 & 1 &
\end{array}\right)_{8}
$$

is a 2 -marked Durfee symbol with 1 st $\operatorname{rank} r_{1}(\tau)=1$ and 2 nd $\operatorname{rank} r_{2}(\tau)=-1$. This is a partition of $87=23+8^{2}$, sum of all entries inside the symbol plus the contribution from the Durfee square.

The difference of the definition of $k$-marked Durfee symbols from that as given in [3, $\S 4]$ is that the largest entries for each index in the top row are written as posts. It is obvious that there is a one to one correspondence between these modified Durfee Symbols and the original ones. To see the other direction of the correspondence, we write $p_{i}$ as $a_{i, m_{i}+1}$ in the top row.

Definition 1.5. Let $\mathcal{S}_{d}^{k}\left(m_{1}, \ldots, m_{d}\right)$ denote the collection of $k$-marked Durfee symbols, the side length of whose durfee squares is $d$, and the total number of appearances of $j$ is exactly $m_{j}$ in each symbol, $j=1, \ldots, d$. This is a subset of $k$-marked Durfee symbols that partition $n=d^{2}+\sum_{j=1}^{d} j m_{j}$.

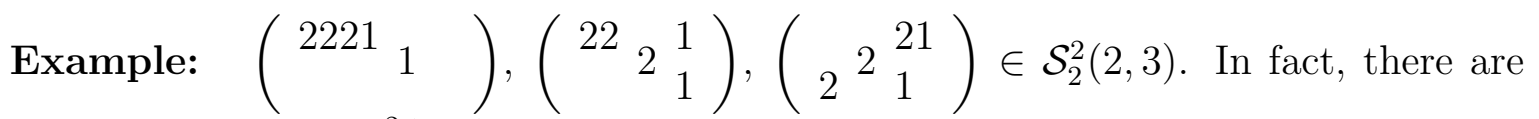
exactly 46 elements in $\mathcal{S}_{2}^{2}(2,3)$, as shown below.

Definition 1.6. Let $\mathcal{S}_{d}^{o k}\left(m_{1}, \ldots, m_{d}\right)$ denote the collection of $k$-marked odd Durfee symbols, the side length of whose durfee squares is $d$, and the total number of appearances of $2 j-1$ is exactly $m_{j}$ in each symbol, $j=1, \ldots, d$. This is a subset of $k$-marked odd Durfee symbols that partition $n=d^{2}-2 d+1+\sum_{j=1}^{d}(2 j-1) m_{j}$.

Observe that $\mathcal{S}_{d}^{1}\left(m_{1}, \ldots, m_{d}\right)=\mathcal{S}_{d}\left(m_{1}, \ldots, m_{d}\right)$, and that $\mathcal{S}_{d}^{o 1}\left(m_{1}, \ldots, m_{d}\right)=$ $\mathcal{S}^{o}{ }_{d}\left(m_{1}, \ldots, m_{d}\right)$.

The generating function of the $k$-marked Durfee symbols when $k \geq 2$ is

$$
\begin{gathered}
R_{k}\left(x_{1}, \ldots, x_{k} ; q\right)= \\
\sum_{\substack{d \geq 1 \\
m_{1}, \ldots, m_{d} \geq 0}} q^{m_{1}+2 m_{2}+\cdots+d m_{d}+d^{2}} \sum_{\tau \in \mathcal{S}_{d}^{k}\left(m_{1}, \ldots, m_{d}\right)} x_{1}^{r_{1}(\tau)} \cdots x_{k}^{r_{k}(\tau)} .
\end{gathered}
$$

When $k=1$, we simply add 1 to the multiple sum. This is because the empty partition is traditionally considered as having rank zero, but the empty partition cannot correspond to any $k$-marked Durfee symbol for $k \geq 2$. Any $k$-marked Durfee symbol $\tau$ partitioning 
$n$ contributes to the term $x_{1}^{r_{1}(\tau)} \cdots x_{k}^{r_{k}(\tau)} q^{n}$. For instance, $\tau$ as above contributes to the term $x_{1} x_{2}^{-1} q^{87}$ in $R_{2}\left(x_{1}, x_{2} ; q\right)$.

The generating function of $k$-marked odd Durfee symbols is

$$
\begin{aligned}
& R_{k}^{o}\left(x_{1}, \ldots, x_{k} ; q\right)=
\end{aligned}
$$

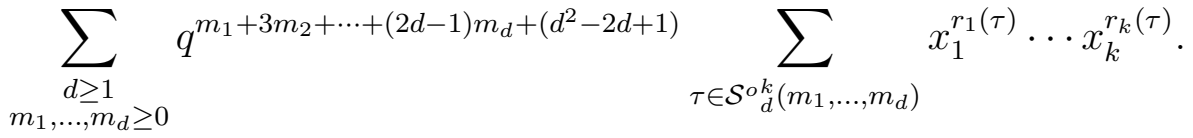

Exponents of $x_{1}, \ldots, x_{k}$ keep track of the 1 st, $\ldots k$ th ranks of symbols, respectively, and the exponent of $q$ keeps track of the number being partitioned.

\section{Basic Constructions}

Lemma 2.1.

$$
\begin{gathered}
\sum_{\tau \in \mathcal{S}_{d}^{1}\left(m_{1}, \ldots, m_{d}\right)} z^{r_{1}(\tau)}=\sum_{\tau \in \mathcal{S}_{d}^{\circ 1}\left(m_{1}, \ldots, m_{d}\right)} z^{r_{1}(\tau)}= \\
=\prod_{j=1}^{d}\left(\frac{z^{m_{j}+1}-z^{-m_{j}-1}}{z-z^{-1}}\right)
\end{gathered}
$$

Proof. A Durfee symbol listed by $\mathcal{S}_{d}\left(m_{1}, \ldots, m_{d}\right)$ looks like

$$
\left(\begin{array}{ccc}
\overbrace{d \ldots d}^{j_{d}} & \overbrace{1 \ldots 1}^{j_{1}} \\
\underbrace{d \ldots d}_{m_{d}-j_{d}} & \cdots & \underbrace{1 \ldots 1}_{m_{1}-j_{1}}
\end{array}\right)_{d}
$$

It has rank $j_{1}-\left(m_{1}-j_{1}\right)+\cdots+j_{d}-\left(m_{d}-j_{d}\right)$. Therefore,

$$
\begin{gathered}
\sum_{\tau \in \mathcal{S}_{d}^{1}\left(m_{1}, \ldots, m_{d}\right)} z^{r_{1}(\tau)}=\sum_{j_{1}=0}^{m_{1}} \ldots \sum_{j_{d}=0}^{m_{d}} z^{j_{1}-\left(m_{1}-j_{1}\right)} \ldots z^{j_{d}-\left(m_{d}-j_{d}\right)} \\
=\left(\sum_{j_{1}=0}^{m_{1}} z^{j_{1}-\left(m_{1}-j_{1}\right)}\right) \ldots\left(\sum_{j_{d}=0}^{m_{d}} z^{j_{d}-\left(m_{d}-j_{d}\right)}\right)=z^{-m_{1}-\ldots-m_{d}}\left(\sum_{j_{1}=0}^{m_{1}} z^{2 j_{1}}\right) \ldots\left(\sum_{j_{d}=0}^{m_{1}} z^{2 j_{d}}\right) \\
=z^{-m_{1}-\ldots-m_{d}}\left(\frac{1-z^{2 m_{1}+2}}{1-z^{2}}\right) \ldots\left(\frac{1-z^{2 m_{d}+2}}{1-z^{2}}\right)=\prod_{j=1}^{d}\left(\frac{z^{m_{j}+1}-z^{-m_{j}-1}}{z-z^{-1}}\right)
\end{gathered}
$$

The proof is identical for odd Durfee symbols, with obvious notational changes. 
Lemma 2.2. For $z \neq w, z \neq 1 / w$,

$$
\begin{gathered}
\sum_{\tau \in \mathcal{S}_{d}^{2}\left(m_{1}, \ldots, m_{d}\right)} z^{r_{1}(\tau)} w^{r_{2}(\tau)}=\sum_{\tau \in \mathcal{S}_{d}^{2}\left(m_{1}, \ldots, m_{d}\right)} z^{r_{1}(\tau)} w^{r_{2}(\tau)}= \\
=\frac{1}{(z+1 / z)-(w+1 / w)}\left(\prod_{j=1}^{d} \frac{z^{m_{j}+1}-z^{-m_{j}-1}}{z-z^{-1}}-\prod_{j=1}^{d} \frac{w^{m_{j}+1}-w^{-m_{j}-1}}{w-w^{-1}}\right)
\end{gathered}
$$

Before we prove the Lemma, we give two corollaries:

\section{Corollary 2.3.}

$$
\begin{aligned}
& R_{2}(z, w ; q)=R_{2}(w, z ; q) \\
& R_{2}^{o}(z, w ; q)=R_{2}^{o}(w, z ; q)
\end{aligned}
$$

This is [3, Corollary 4] and[3, Corollary 23] for $k=2$.

Proof. Immediate by inspection of (1.1), (1.2) and (2.3).

\section{Corollary 2.4 .}

$$
\begin{aligned}
& R_{2}(z, w ; q)=\frac{R_{1}(z ; q)-R_{1}(w ; q)}{(z+1 / z)-(w+1 / w)} \\
& R_{2}^{o}(z, w ; q)=\frac{R_{1}^{o}(z ; q)-R_{1}^{o}(w ; q)}{(z+1 / z)-(w+1 / w)}
\end{aligned}
$$

This is [3, Theorem 7] and [3, Theorem 25] for $k=2$.

Proof. Combine (1.1), and (2.1) to obtain (2.4); and (1.2) and (2.3) to obtain (2.5).

proof of Lemma 2.2. A 2-marked Durfee symbol listed by $\mathcal{S}_{d}^{2}\left(m_{1}, \ldots, m_{d}\right)$ looks like

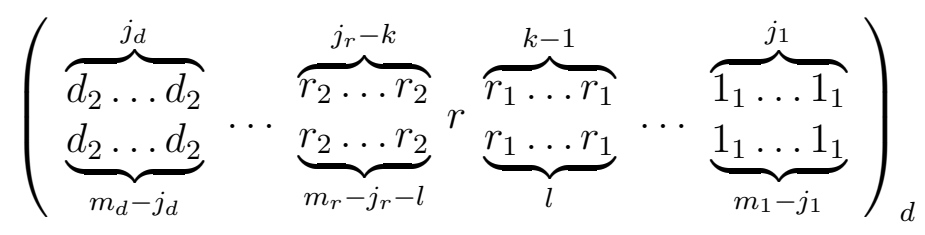

where the post is $r$, and subscripts indicate the mark. We require that $m_{r}, j_{r}, k \geq 1$ by the definition of marked Durfee symbols. However, once we compute the sum, $m_{r} \geq 1$ will be implied. The contribution to first or second rank due to entries $\neq r$ are found using (2.1).

The contribution to both the first and the second ranks due to $r \mathrm{~s}$ in

$$
\sum_{\tau \in \mathcal{S}_{d}^{2}\left(m_{1}, \ldots, m_{d}\right)} z^{r_{1}(\tau)} w^{r_{2}(\tau)}
$$


when the post is $r$ is given by:

$$
\begin{aligned}
& \sum_{j_{r}=1}^{m_{r}} \sum_{k=1}^{j_{r}} \sum_{l=0}^{m_{r}-j_{r}} z^{(k-1)-l} w^{\left(j_{r}-k\right)-\left(m_{r}-j_{r}-l\right)} \\
& =\frac{w^{-m_{r}}}{z} \sum_{j_{r}=1}^{m_{r}} w^{2 j_{r}} \sum_{k=1}^{j_{r}}\left(\frac{z}{w}\right)^{k} \sum_{l=0}^{m_{r}-j_{r}}\left(\frac{w}{z}\right)^{l} \\
& =\frac{w^{-m_{r}}}{z} \sum_{j_{r}=1}^{m_{r}} w^{2 j_{r}}\left(\frac{\frac{z}{w}-\left(\frac{z}{w}\right)^{j_{r}+1}}{1-\frac{z}{w}}\right)\left(\frac{1-\left(\frac{w}{z}\right)^{m_{r}-j_{r}+1}}{1-\frac{w}{z}}\right) \\
& =\frac{w^{-m_{r}-1}}{(1-z / w)(1-w / z)} \sum_{j_{r}=1}^{m_{r}} w^{2 j_{r}}-(w z)^{j_{r}}-\left(\frac{w}{z}\right)^{m_{r}+1}(w z)^{j_{r}}+\left(\frac{w}{z}\right)^{m_{r}+1} z^{2 j_{r}} \\
& =\frac{w^{-m_{r}-1} z^{-m_{r}-1}}{(1-z / w)(1-w / z)}\left[z^{m_{r}+1} \frac{w^{2}-w^{2 m_{r}+2}}{1-w^{2}}\right. \\
& \left.-\left(w^{m_{r}+1}+z^{m_{r}+1}\right) \frac{w z-(w z)^{m_{r}+1}}{1-w z}+w^{m_{r}+1} \frac{z^{2}-z^{2 m_{r}+2}}{1-z^{2}}\right] \\
& =\frac{1}{(1-z / w)(1-w / z)}\left[w^{-m_{r}-1}\left(\frac{w^{2}}{1-w^{2}}-\frac{w z}{1-w z}\right)+w^{m_{r}+1}\left(\frac{1}{1-w z}-\frac{1}{1-w^{2}}\right)\right. \\
& \left.z^{-m_{r}-1}\left(\frac{z^{2}}{1-z^{2}}-\frac{w z}{1-w z}\right)+z^{m_{r}+1}\left(\frac{1}{1-w z}-\frac{1}{1-w^{2}}\right)\right] \\
& =\frac{1}{(z+1 / z)-(w+1 / w)}\left[\frac{z^{m_{r}+1}-z^{-m_{r}-1}}{z-z^{-1}}-\frac{w^{m_{r}+1}-w^{-m_{r}-1}}{w-w^{-1}}\right]
\end{aligned}
$$

Now we sum over $r=1, \ldots, d$ :

$$
\begin{gathered}
\frac{1}{(z+1 / z)-(w+1 / w)} \sum_{r=1}^{d} \prod_{j=r+1}^{d}\left(\frac{w^{m_{j}+1}-w^{-m_{j}-1}}{w-w^{-1}}\right) \\
\times\left(\frac{z^{m_{r}+1}-z^{-m_{r}-1}}{z-z^{-1}}-\frac{w^{m_{r}+1}-w^{-m_{r}-1}}{w-w^{-1}}\right) \prod_{j=1}^{r-1}\left(\frac{z^{m_{j}+1}-z^{-m_{j}-1}}{z-z^{-1}}\right)
\end{gathered}
$$

Upon expansion of the middle factor the sum telescopes and the result follows.

Proposition 2.5.

$$
\begin{gathered}
\# \mathcal{S}_{d}^{k+1}\left(m_{1}, \ldots, m_{d}\right)=\sum_{\tau \in \mathcal{S}_{d}^{k+1}\left(m_{1}, \ldots, m_{d}\right)} 1 \\
=\sum_{k_{1}+\ldots+k_{d}=k}\left(\begin{array}{c}
m_{1}+k_{1}+1 \\
2 k_{1}+1
\end{array}\right) \ldots\left(\begin{array}{c}
m_{d}+k_{d}+1 \\
2 k_{d}+1
\end{array}\right)
\end{gathered}
$$


Proof. Assume that for some $r$ between 1 and $d$, there are exactly $m_{r} r$ in a Durfee Symbol, $j$ of which are in the top row, and $m_{r}-j$ are in the bottom row. To introduce $k_{r}$ posts (so as to make $r \mathrm{~s}\left(k_{r}+1\right)$-marked), following the definitions in [3]; we need to choose $k_{r}$ of the $j$ elements in the top row, none repeated; and $k_{r}$ possibly repeated posts from the $m_{r}-j+1$ posts between the entries in the bottom row. There are

$$
\left(\begin{array}{c}
j \\
k_{r}
\end{array}\right)\left(\begin{array}{c}
m_{r}-j+1+k_{r}-1 \\
k_{r}
\end{array}\right)
$$

ways to do this $[10, \S 1.1]$. Upon summing over $j=0, \ldots, m_{r}$ using [7, eq.(5.26)], we have

$$
\left(\begin{array}{c}
m_{r}+k_{r}+1 \\
2 k_{r}+1
\end{array}\right)
$$

Then, to make the whole symbol $k+1$-marked, we need to choose a total of $k$ posts, $k_{r}$ of them in $r \mathrm{~s}$ in the Durfee symbol. This gives us the asserted sum.

The symmetrized kth moment function is defined [3, eq.(1.13)] as

$$
\eta_{k}(n)=\sum_{m=-\infty}^{\infty}\left(\begin{array}{c}
m+\left\lfloor\frac{k-1}{2}\right\rfloor \\
k
\end{array}\right) N(m, n)
$$

where $N(m, n)$ is the number of partitions of $n$ with rank $m$.

\section{Proposition 2.6.}

$$
\begin{aligned}
& \sum_{\tau \in \mathcal{S}_{d}\left(m_{1}, \ldots, m_{d}\right)}\left(\begin{array}{c}
r(\tau)+k-1 \\
2 k
\end{array}\right)
\end{aligned}
$$

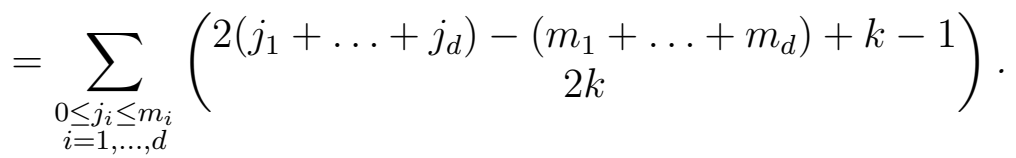

Proof. A Durfee symbol having $j_{r} r \mathrm{~s}$ in the top row, $m_{r}-j_{r} r \mathrm{~s}$ in the bottom row for $r=1, \ldots, d$ has rank $2\left(j_{1}+\ldots+j_{d}\right)-\left(m_{1}+\ldots+m_{d}\right)$.

\section{Applications}

The line of reasoning in (2.2) yields

$$
\sum_{\tau \in \mathcal{S}_{d}\left(m_{1}, \ldots, m_{d}\right)}(-1)^{r(\tau)}=(-1)^{m_{1}+\ldots+m_{d}}\left(m_{1}+1\right) \ldots\left(m_{d}+1\right)
$$

which is the number of partitions in $\mathcal{S}_{d}\left(m_{1}, \ldots, m_{d}\right)$ weighted by $(-1)^{\mathrm{rank}}$. Note that the parity of rank is invariant in $\mathcal{S}_{d}\left(m_{1}, \ldots, m_{d}\right)$. 
A more interesting computation is that

$$
\sum_{\tau \in \mathcal{S}_{d}\left(m_{1}, \ldots, m_{d}\right)} i^{r(\tau)}=\Im\left(i^{m_{1}+1}\right) \ldots \Im\left(i^{m_{d}+1}\right) .
$$

This product vanishes unless all $m_{j}$ s are even, and it is $(-1)^{\left(m_{1}+\ldots+m_{d}\right) / 2}$ then. We interpret that as follows. $\mathcal{S}_{d}\left(m_{1}, \ldots, m_{d}\right)$ is annihilated in $R_{1}(i, q)$ unless it contains a self conjugate partition, and self conjugate partitions are given the factor

$$
(-1)^{\text {number of parts below the Durfee square }} \text {. }
$$

The formulae derived in Lemma 2.1 prove [3, eq.s (13.1)-(13.4)], which follow. Of course, the equations are mere substitutions in the generating functions in [3]. The point is that the following identities are proven without having the exact formula of the generating function of the $k$-marked Durfee symbols (or $k$-marked odd Durfee symbols).

$$
\begin{aligned}
& R_{1}(-1 ; q)=\sum_{n \geq 0} \frac{q^{n^{2}}}{(-q ; q)_{n}^{2}} \\
& R_{1}(i ; q)=\sum_{n \geq 0} \frac{q^{n^{2}}}{\left(-q^{2} ; q^{2}\right)_{n}} \\
& R_{1}^{o}(-1 ; q)=\sum_{n \geq 0} \frac{q^{2 n^{2}+2 n+1}}{\left(q ; q^{2}\right)_{n+1}^{2}} \\
& R_{1}^{o}(i ; q)=\sum_{n \geq 0} \frac{q^{2 n^{2}+2 n+1}}{\left(-q^{2} ; q^{4}\right)_{n+1}}
\end{aligned}
$$

It is also straightforward combine Lemma 2.2, and equations (3.1)-(3.4) to prove [3, eq.s (13.7) and (13.9)], which follow.

$$
\begin{gathered}
R_{2}(i,-1 ; q)=\frac{1}{2}\left(\sum_{n \geq 0} \frac{q^{n^{2}}}{\left(-q^{2} ; q^{2}\right)_{n}}-\sum_{n \geq 0} \frac{q^{n^{2}}}{(-q ; q)_{n}^{2}}\right) \\
R_{2}^{o}(i,-1 ; q)=\frac{1}{2}\left(\sum_{n \geq 0} \frac{q^{2 n^{2}+2 n+1}}{\left(-q^{2} ; q^{4}\right)_{n+1}}+\sum_{n \geq 0} \frac{q^{2 n^{2}+2 n+1}}{\left(-q ; q^{2}\right)_{n+1}^{2}}\right)
\end{gathered}
$$

Although the idea of the proofs was to show that both sides generate $\mathcal{S}_{d}\left(m_{1}, \ldots, m_{d}\right)$ with the same factor, it is not hard to give a combinatorial description of the partitions enumerated by the right hand side of, for instance (3.5). Self conjugate partitions are annihilated if the number of parts below the Durfee square is even. Self conjugate partitions with an odd number of parts below the Durfee square gain weight 2, which is then halved. For conjugate pairs of distinct partitions, exactly one partition is counted 
by sign depending on the number of parts appearing in its Durfee symbol. We can even lexicographically order the rows in the Durfee symbol to make the choice.

It is possible to extend the results to obtain [3, Theorem 7], and hence or otherwise prove that $R_{k}\left(x_{1}, \ldots, x_{k} ; q\right)$ is symmetric in $x_{1}, \ldots, x_{k}$. One needs to refine the definition of $\mathcal{S}_{d}^{k}\left(m_{1}, \ldots, m_{d}\right)$, and deal with two adjacent variables at once.

Definition 3.1. Given $k$ and $i$ such that $1 \leq i \leq k$, and a $k$-marked Durfee symbol $\tau$ (respectively, a $k$-marked odd Durfee symbol $\tau^{o}$ ), and a non-negative integer $g$, Let $\mathcal{S}_{(k, i)}^{k+g}(\tau)$ (respectively, $\mathcal{S}_{(k, i)}^{o k+g}\left(\tau^{o}\right)$ ) denote the collection of $(k+g)$-marked Durfee symbols (respectively, $k+g$-marked odd Durfee symbols) which have the same number of occurrences of each part, the same side length of Durfee squares, and such that

i) the $k+g, k+g-1, \ldots, i+g+1, i-1, i-2, \ldots, 1$-marked double arrays of the symbols in the equivalence class are identical to the $k, k-1, \ldots, i+1, i-1, i-2, \ldots, 1$-marked double arrays of $\tau$ (resp. $\left.\tau^{o}\right)$, respectively,

ii) the $(k+g-1)$ th $,(k+g-2) t h, \ldots,(i+g)$ th, $(i-1)$ th,$(i-2)$ th $, \ldots, 1$ st posts of the symbols in the equivalence class are identical to the $(k-1) t h,(k-2) t h, \ldots, 1$ st posts of $\tau$ (resp. $\left.\tau^{o}\right)$, respectively.

Verbally, given a $k$-marked Durfee symbol $\tau$ with a specified mark $i(1 \leq i \leq k)$, we rearrange the $i$-marked parts in $\tau$ in every possible way. Introducing $g$ new posts hence making the symbols $k+g$ marked is also allowed.

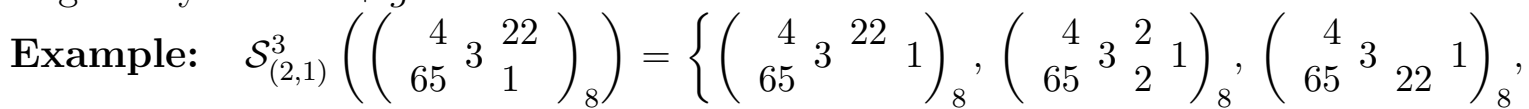

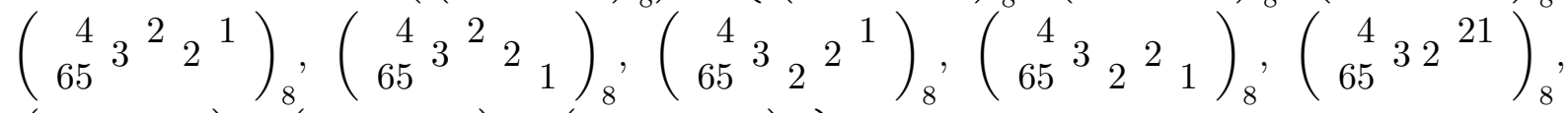

$$
\begin{aligned}
& \left.\left(\begin{array}{rrrr}
4 & 3 & 2 \\
65 & 2 & 1
\end{array}\right)_{8},\left(\begin{array}{rrrr}
4 & 3 & 2 & 1 \\
65 & & 2
\end{array}\right)_{8},\left(\begin{array}{rrrr}
4 & 3 & 2 \\
65 & & 21
\end{array}\right)_{8}\right\} \text {. }
\end{aligned}
$$

Lemma 3.2. Let $\tau$ be a k-marked Durfee symbol with $(i-1)$ th and ith posts $p_{i-1}$ and $p_{i}$. Let $m_{p_{i-1}}, m_{p_{i-1}}+1, \ldots, m_{p_{i}}$ be the number of occurrences of $p_{i-1}, p_{i-1}+1, \ldots, p_{i}$, respectively, in the ith double array of $\tau$. Then,

$$
\sum_{\varrho \in \mathcal{S}_{(k, i)}^{k}(\tau)} x_{i}^{r_{i}(\varrho)}=\prod_{j=p_{i-1}}^{p_{i}} \frac{x_{i}^{m_{j}+1}-x_{i}^{-m_{j}-1}}{x_{i}-x_{i}^{-1}}
$$

Proof. A symbol listed by $\mathcal{S}_{(k, i)}(\tau)$ looks like

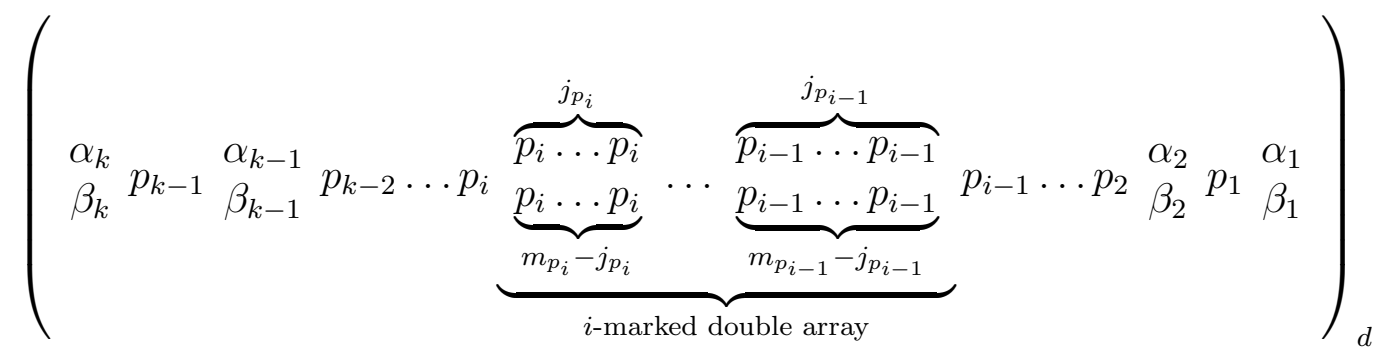


and it contributes to the exponent of $x_{i}$ in the appropriate term in the generating function of $k$-marked Durfee symbols with

$$
x_{i}^{j_{p_{i}}-\left(m_{p_{i}}-j_{p_{i}}\right)} \ldots x_{i}^{j_{p_{i-1}}-\left(m_{p_{i-1}}-j_{p_{i-1}}\right)} .
$$

Then the computations in (2.2) apply with the obvious notational changes.

Lemma 3.3. Let $\tau$ be a k-marked Durfee symbol with $(i-1)$ th and ith posts $p_{i-1}$ and $p_{i}$. Let $m_{p_{i-1}}, m_{p_{i-1}}+1, \ldots, m_{p_{i}}$ be the number of occurrences of $p_{i-1}, p_{i-1}+1, \ldots, p_{i}$, respectively, in the ith double array of $\tau$. Then,

$$
\begin{gathered}
\sum_{\varrho \in \mathcal{S}_{(k, i)}^{k+1}(\tau)} x_{i}^{r_{i}(\varrho)} x_{i+1}^{r_{i+1}(\varrho)}= \\
\frac{1}{\left(x_{i}-1 / x_{i}\right)-\left(x_{i-1}-1 / x_{i-1}\right)}\left(\prod_{j=p_{i-1}}^{p_{i}} \frac{x_{i}^{m_{j}+1}-x_{i}^{-m_{j}-1}}{x_{i}-x_{i}^{-1}}-\prod_{j=p_{i-1}}^{p_{i}} \frac{x_{i-1}^{m_{j}+1}-x_{i-1}^{-m_{j}-1}}{x_{i-1}-x_{i-1}^{-1}}\right)
\end{gathered}
$$

Proof. We will choose a post among the entries in the $i$ th double array in $\tau$, and make it into a $(k+1)$-marked Durfee symbol. We will then keep track of the $i$ th rank as the exponent of $x_{i}$, and of the $(i+1)$ th rank as the exponent of $x_{i+1}$. The other double arrays and posts are kept fixed by definition of $\mathcal{S}_{(k, i)}(\tau)$, the only portion of $\tau$ that can be played around with is the $i$ th double array. $\tau$ after the choice of an extra post will look like

$$
\begin{aligned}
& \left(\begin{array}{ccc}
\alpha_{k} & \alpha_{k-1} \\
\beta_{k} & p_{k-1} & \beta_{k-1} \\
& p_{k-2} \ldots p_{i}
\end{array}\right.
\end{aligned}
$$

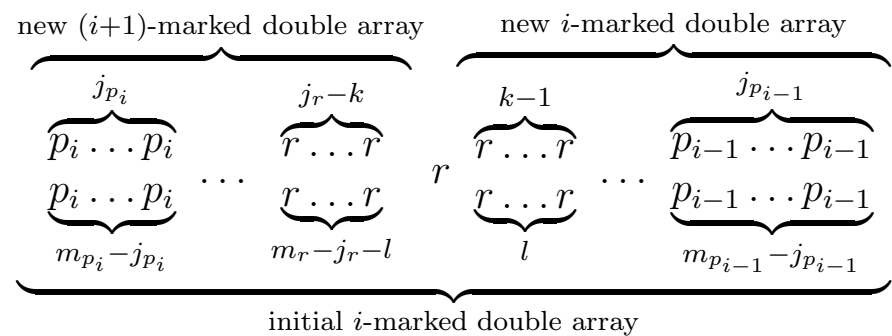

$$
\begin{aligned}
& \left.p_{i-1} \ldots p_{2} \begin{array}{ccc}
\alpha_{2} & \alpha_{1} \\
\beta_{2} & p_{1} & \beta_{1}
\end{array}\right)_{d}
\end{aligned}
$$

for some $r$ between $p_{i-1}$ and $p_{i}$. At this point, we make the substitutions

$$
\begin{aligned}
p_{k+1} & \leftarrow p_{k} \\
p_{k} & \leftarrow p_{k-1} \\
& \vdots \\
p_{i+1} & \leftarrow p_{i} \\
p_{i} & \leftarrow r
\end{aligned}
$$

along with the corresponding re-marking of $\alpha \mathrm{s}$ and $\beta \mathrm{s}$ in between these new posts, so that the exponent of $x_{j}$ now keeps track of $j$ th rank for $j=1, \ldots,(k+1)$. 
The $i$ th rank, as the exponent of $x_{i}$ is

$$
\left(j_{i-1}-\left(m_{i-1}-j_{i-1}\right)\right)+\ldots+\left(j_{r-1}-\left(m_{r-1}-j_{r-1}\right)\right)+(k-l)
$$

and the $(i+1)$ th rank as the exponent of $x_{i+1}$ is

$$
\left(j_{r}-k-\left(m_{r}-j_{r}-l\right)\right)\left(j_{r+1}-\left(m_{r+1}-j_{r+1}\right)\right)+\ldots+\left(j_{i}-\left(m_{i}-j_{i}\right)\right)
$$

Then, the computations in (2.3) carry over with appropriate notational changes.

Corollary 3.4. $R_{k}\left(x_{1}, \ldots, x_{k} ; q\right)$ is symmetric in $x_{1}, \ldots, x_{k}$.

This is [3, Corollary 4].

Proof. For any fixed $i=1, \ldots, k$,

$$
\begin{gathered}
R_{k+1}\left(x_{1}, \ldots, x_{k+1} ; q\right)=\sum_{\substack{d \geq 1 \\
m_{1}, \ldots, m_{d} \geq 0}} q^{m_{1}+2 m_{2} \ldots+d m_{d}+d^{2}} \\
\times \sum_{\tau \in \mathcal{S}_{d}^{k}\left(m_{1}, \ldots, m_{d}\right)} x_{1}^{r_{1}(\tau)} \cdots x_{i-1}^{r_{i-1}(\tau)} x_{i+2}^{r_{i+1}(\tau)} \cdots x_{k+1}^{r_{k}(\tau)} \sum_{\varrho \in \mathcal{S}_{(k, i)}^{k+1}(\tau)} x_{i}^{r_{i}(\varrho)} x_{i+1}^{r_{i+1}(\varrho)} .
\end{gathered}
$$

Because any $(k+1)$-marked Durfee symbol $\varrho$ could be thought of as belonging to some equivalence class $\mathcal{S}_{(k, i)}^{k+1}(\tau)$, and no $\varrho$ corresponds to two distinct equivalence classes at once for any fixed $i$. Also, when $\varrho \in \mathcal{S}_{(k, i)}^{k+1}(\tau)$, the 1 st, 2 nd, $\ldots,(i-1)$ th, $(i+1)$ th, $\ldots, k$ th ranks of $\tau$ are the same as the 1 st, 2 nd, .., $(i-1)$ th, $(i+2)$ th, .., $(k+1)$ th ranks of $\varrho$, respectively, by definition.

Thus, by Lemma 3.3 we see that

$$
R_{k+1}\left(x_{1}, \ldots, x_{i}, x_{i+1}, \ldots, x_{k}\right)=R_{k+1}\left(x_{1}, \ldots, x_{i-1}, x_{i+1}, x_{i}, x_{i+2}, \ldots, x_{k}\right)
$$

Then, we use the well known fact that pairwise switching of adjacent symbols (in this case, $x_{j} \mathrm{~s}$ ) generate the symmetric group on $k+1$ letters. Upon $k \rightarrow k-1$, the Corollary follows.

Corollary 3.5. For $k \geq 2$, and $x_{i} \neq x_{j}, x_{i} \neq 1 / x_{j}$ for $i \neq j, i, j=1, \ldots, k$,

$$
R_{k}\left(x_{1}, \ldots, x_{k} ; q\right)=\sum_{i=1}^{k} \frac{R_{1}\left(x_{i} ; q\right)}{\prod_{j=1, j \neq i}^{k}\left[\left(x_{i}+\frac{1}{x_{i}}\right)-\left(x_{j}+\frac{1}{x_{j}}\right)\right]}
$$

This is [3, Theorem 7]. The proof in this context would be identical to the one given in [4], which is mathematical induction on $k$. The base case is given by Corollary 2.4, and the inductive step is provided by Lemma 3.3 along with (3.9).

\section{Theorem 3.6.}

$$
R_{k+1}(1, \ldots, 1 ; q)=\sum_{n \geq 0} \eta_{2 k}(n) q^{n}
$$


This is [3, Corollary 5].

In other words, $\eta_{2 k}(n)$ counts the number of $(k+1)$-marked Durfee symbols associated to $n$ [3, Corollary 13].

Proof. It suffices to establish

$$
\begin{gathered}
\sum_{k_{1}+\ldots+k_{d}=k}\left(\begin{array}{c}
m_{1}+k_{1}+1 \\
2 k_{1}+1
\end{array}\right) \ldots\left(\begin{array}{c}
m_{d}+k_{d}+1 \\
2 k_{d}+1
\end{array}\right) \\
=\sum_{\substack{0 \leq j_{i} \leq m_{i} \\
i=1, \ldots, d}}\left(\begin{array}{c}
2\left(j_{1}+\ldots+j_{d}\right)-\left(m_{1}+\ldots+m_{d}\right)+k-1 \\
2 k
\end{array}\right) .
\end{gathered}
$$

Since, by Proposition 2.5

$$
\begin{gathered}
\sum_{n \geq 0} \eta_{2 k}(n) q^{n}=\sum_{\substack{d \geq 1 \\
m_{1}, \ldots, m_{d} \geq 0}} q^{m_{1}+2 m_{2}+\ldots+d m_{d}+d^{2}} \sum_{\tau \in \mathcal{S}_{d}\left(m_{1}, \ldots, m_{d}\right)}\left(\begin{array}{c}
r(\tau)+k-1 \\
2 k
\end{array}\right) \\
=\sum_{\substack{d \geq 1 \\
m_{1}, \ldots, m_{d} \geq 0}} q^{m_{1}+2 m_{2}+\ldots+d m_{d}+d^{2}} \sum_{\substack{0 \leq j_{i} \leq m_{i} \\
i=1, \ldots, d}}\left(\begin{array}{c}
2\left(j_{1}+\ldots+j_{d}\right)-\left(m_{1}+\ldots+m_{d}\right)+k-1 \\
2 k
\end{array}\right),
\end{gathered}
$$

and by Proposition 2.6 and (1.1),

$$
\begin{aligned}
& R_{k+1}(1, \ldots, 1 ; q)=\sum_{\substack{d \geq 1 \\
m_{1}, \ldots, m_{d} \geq 0}} q^{m_{1}+2 m_{2}+\ldots+d m_{d}+d^{2}} \sum_{\tau \in \mathcal{S}_{d}^{k}\left(m_{1}, \ldots, m_{d}\right)} 1 \\
& =\sum_{\substack{d \geq 1 \\
m_{1}, \ldots, m_{d} \geq 0}} q^{m_{1}+2 m_{2}+\ldots+d m_{d}+d^{2}} \sum_{k_{1}+\ldots+k_{d}=k}\left(\begin{array}{c}
m_{1}+k_{1}+1 \\
2 k_{1}+1
\end{array}\right) \ldots\left(\begin{array}{c}
m_{d}+k_{d}+1 \\
2 k_{d}+1
\end{array}\right) .
\end{aligned}
$$

We use mathematical induction on $d$. For $d=1$ we have:

$$
\left(\begin{array}{c}
m+k+1 \\
2 k+1
\end{array}\right)=\sum_{j=0}^{m}\left(\begin{array}{c}
2 j-m+k-1 \\
2 k
\end{array}\right)
$$

Now, for $k=0$, we have $m+1=m+1$. For $k$ positive,

$$
\begin{gathered}
\sum_{j=0}^{m}\left(\begin{array}{c}
2 j-m+k-1 \\
2 k
\end{array}\right)=\sum_{0 \leq j \leq\lfloor m / 2\rfloor}\left(\begin{array}{c}
2 j-m+k-1 \\
2 k
\end{array}\right)+\sum_{\lfloor m / 2\rfloor<j \leq m}\left(\begin{array}{c}
2 j-m+k-1 \\
2 k
\end{array}\right) \\
=\sum_{0 \leq j \leq\lfloor m / 2\rfloor}\left(\begin{array}{c}
m-2 j+k \\
2 k
\end{array}\right)+\sum_{\lfloor m / 2\rfloor<j \leq m}\left(\begin{array}{c}
2 j-m+k-1 \\
2 k
\end{array}\right)=\sum_{j=0 \text { or } 1}^{m}\left(\begin{array}{c}
j+k \\
2 k
\end{array}\right) \\
=\left(\begin{array}{c}
m+k+1 \\
2 k+1
\end{array}\right)
\end{gathered}
$$


where we negated the upper index in the first half of the sum and used (5.10) in [7]. This provides the base case.

Assuming for some $d$, after applying the inductive hypothesis, and upon substituting

$$
\begin{aligned}
2\left(j_{1}+\ldots+j_{d}\right) & -\left(m_{1}+\ldots+m_{d}\right) \leftarrow N, \\
m_{d+1} & \leftarrow m, \\
j_{d+1} & \leftarrow d,
\end{aligned}
$$

we are down to proving

$$
\sum_{i=0}^{k}\left(\begin{array}{c}
m+(k-i)+1 \\
2(k-i)+1
\end{array}\right)\left(\begin{array}{c}
N+i-1 \\
2 i
\end{array}\right)=\sum_{j=0}^{m}\left(\begin{array}{c}
N+2 j-m+k-1 \\
2 k
\end{array}\right)
$$

for $k, m \geq 0$, and $N \in \mathbb{Z}$.

In fact, it suffices to establish the result for non-negative $N$, since

$$
\left(\begin{array}{c}
N+i-1 \\
2 i
\end{array}\right)=(-1)^{2 i}\left(\begin{array}{c}
(2 i-1)-(N+i-1) \\
2 i
\end{array}\right)=\left(\begin{array}{c}
(1-N)+i-1 \\
2 i
\end{array}\right)
$$

and

$$
\begin{gathered}
\left(\begin{array}{c}
N+2 j-m+k-1 \\
2 k
\end{array}\right)=\left(\begin{array}{c}
(2 k-1)-(N+2 j-m+k-1) \\
2 k
\end{array}\right) \\
=\left(\begin{array}{c}
(1-N)+m-2 j+k-1 \\
2 k
\end{array}\right)
\end{gathered}
$$

using [7, eq.(5.14)]. For the latter derivation, we are also allowed to substitute $j \leftarrow m-j$ under the sum over $0 \leq j \leq m$.

For integral $k, m, N \geq 0$, we define

$$
\begin{gathered}
L_{e}(N, k)=\sum_{i=0}^{k}\left(\begin{array}{c}
m+(k-i)+1 \\
2(k-i)+1
\end{array}\right)\left(\begin{array}{c}
N+i-1 \\
2 i
\end{array}\right) \\
R_{e}(N, k)=\sum_{j=0}^{m}\left(\begin{array}{c}
N+2 j-m+k-1 \\
2 k
\end{array}\right) \\
L_{o}(N, k)=\sum_{i=0}^{k}\left(\begin{array}{c}
m+(k-i)+1 \\
2(k-i)+1
\end{array}\right)\left(\begin{array}{c}
N+i-1 \\
2 i-1
\end{array}\right) \\
R_{o}(N, k)=\sum_{j=0}^{m}\left(\begin{array}{c}
N+2 j-m+k-1 \\
2 k-1
\end{array}\right)
\end{gathered}
$$

Then, using the basic recurrence for binomial coefficients, we obtain

$$
L_{e}(N, k)=\sum_{i=0}^{k}\left(\begin{array}{c}
m+(k-i)+1 \\
2(k-i)+1
\end{array}\right)\left(\left(\begin{array}{c}
(N-1)+i-1 \\
2 i
\end{array}\right)+\left(\begin{array}{c}
(N-1)+i-1 \\
2 i-1
\end{array}\right)\right)
$$




$$
=L_{e}(N-1, k)+L_{o}(N-1, k)
$$

and

$$
\begin{gathered}
L_{o}(N, k)=\sum_{i=0}^{k}\left(\begin{array}{c}
m+(k-i)+1 \\
2(k-i)+1
\end{array}\right)\left(\left(\begin{array}{c}
N+i-2 \\
2 i-1
\end{array}\right)+\left(\begin{array}{c}
N+i-2 \\
2 i-2
\end{array}\right)\right) \\
=L_{o}(N-1, k)+\sum_{i=0}^{k-1}\left(\begin{array}{c}
m+((k-1)-i)+1 \\
2((k-1)-i)+1
\end{array}\right)\left(\begin{array}{c}
N+i-1 \\
2 i
\end{array}\right)=L_{o}(N-1, k)+L_{e}(N, k-1)
\end{gathered}
$$

Similar reasoning shows that

$$
\begin{gathered}
R_{e}(N, k)=\sum_{j=0}^{m}\left(\left(\begin{array}{c}
N-1+2 j-m+k-1 \\
2 k
\end{array}\right)+\left(\begin{array}{c}
N-1+2 j-m+k-1 \\
2 k-1
\end{array}\right)\right) \\
=R_{e}(N-1, k)+R_{o}(N-1, k)
\end{gathered}
$$

and that

$$
\begin{gathered}
R_{o}(N, k)=\sum_{j=0}^{m}\left(\left(\begin{array}{c}
N-1+2 j-m+k-1 \\
2 k-1
\end{array}\right)+\left(\begin{array}{c}
N+2 j-m+(k-1)-1 \\
2 k-2
\end{array}\right)\right) \\
=R_{o}(N-1, k)+R_{e}(N, k-1)
\end{gathered}
$$

Thus, the $L \mathrm{~s}$ satisfy the same pair of recurrences as the $R \mathrm{~s}$. The following base cases then establish

$$
L_{e}(N, k)=R_{e}(N, k)
$$

which is (3.12), and

$$
L_{o}(N, k)=R_{o}(N, k)
$$

which is a side result.

Please note that

$$
L_{e}(0, k)=\left(\begin{array}{c}
m+k+1 \\
2 k+1
\end{array}\right)=\sum_{j=0}^{m}\left(\begin{array}{c}
2 j-m+k-1 \\
2 k
\end{array}\right)=R_{e}(0, k)
$$

as shown above in the case $d=1$. Also,

$$
\begin{aligned}
& L_{e}(N, 0)=m+1=R_{e}(N, 0) \\
& L_{o}(N, 0)=m+1=R_{o}(N, 0)
\end{aligned}
$$

is immediate.

$$
L_{o}(0, k)=0
$$

and

$$
R_{o}(0, k)=\sum_{j=0}^{m}\left(\begin{array}{c}
2 j-m+k-1 \\
2 k-1
\end{array}\right)
$$




$$
=\sum_{0 \leq j \leq\lfloor m / 2\rfloor}\left(\begin{array}{c}
2 j-m+k-1 \\
2 k-1
\end{array}\right)+\sum_{\lceil m / 2\rceil \leq j \leq m}\left(\begin{array}{c}
2 j-m+k-1 \\
2 k-1
\end{array}\right)
$$

Now we substitute $j \leftarrow m-j$ in the second sum, and negate the upper index

$$
\begin{aligned}
& =\sum_{0 \leq j \leq\lfloor m / 2\rfloor}\left(\begin{array}{c}
2 j-m+k-1 \\
2 k-1
\end{array}\right) \\
& +\sum_{0 \leq j \leq m-\lceil m / 2\rceil}(-1)^{2 k-1}\left(\begin{array}{c}
((2 k-1)-1)-(m-2 j+k-1) \\
2 k-1
\end{array}\right) \\
& =\sum_{0 \leq j \leq\lfloor m / 2\rfloor}\left(\begin{array}{c}
2 j-m+k-1 \\
2 k-1
\end{array}\right)-\sum_{0 \leq j \leq\lfloor m / 2\rfloor}\left(\begin{array}{c}
2 j-m+k-1 \\
2 k-1
\end{array}\right)=0
\end{aligned}
$$

as desired.

The proof of (3.12) above is given by Andrews [private communication]. The original proof is a double induction on $m$ and $k$.

The side result of the double recursion in the preceding proof is

$$
\sum_{i=1}^{k}\left(\begin{array}{c}
m+(k-i)+1 \\
2(k-i)+1
\end{array}\right)\left(\begin{array}{c}
N+i-1 \\
2 i-1
\end{array}\right)=\sum_{j=0}^{m}\left(\begin{array}{c}
N+2 j-m+k-1 \\
2 k-1
\end{array}\right)
$$

In the context of $k$-marked Durfee symbols, this would be related to moments of odd ranks, which are zero, as shown in [3, Theorem 1].

\section{Conclusion and Further Research}

As stated in the introduction, Ji [8] solved many more open problems listed in [3] using generating function techniques. The aim of this paper was to use more elementary methods on conveniently chosen sets of objects.

The idea of proofs may be applied to other partition identities. The challenge is to choose the appropriate set of objects for the identity at hand. For example, $\mathcal{S}_{d}\left(m_{1}, \ldots, m_{d}\right)$ fails to explain the Rogers-Ramanujan identities [9] in the sense that almost no $\mathcal{S}_{d}\left(m_{1}, \ldots, m_{d}\right)$ is assigned the same factor by the series and the product side. Yet, the empirical evidence suggests that the distribution of factors among $\mathcal{S}_{d}\left(m_{1}, \ldots, m_{d}\right) \mathrm{s}$ corresponding to the same number $n=d^{2}+\sum j m_{j}$ by the product side follows some pattern.

A computer assisted proof of (3.11) is unknown to the author. This will not only enhance the understanding of the identity, but also possibly lead to the discovery of similar identities. A $q$-analog of (3.11) along with combinatorial interpretation may be another reserach problem. The technique Andrews developed in [2] for producing $q$-analogs of binomial coefficient identities does not yield well-poised or balanced $q$-series [6], so the standard hieararchy of $q$-series identities does not readily help. 


\section{References}

[1] G.E. Andrews, The Theory of Partitions, Addison-Wesley Publishing Company, Inc., Reading, MA, 1976. Reissued: Cambridge University Press, 1998.

[2] G.E. Andrews, Applications of basic hypergeometric functions, SIAM Rev., 16(4):441-484, 1974.

[3] G.E. Andrews, Partitions, Durfee symbols, and the Atkin-Garvan moments of ranks, Inventiones Mathematicae, 169:37-73, 2007.

[4] C. Boulet and K. Kurşungöz, Symmetry of $k$-marked Durfee symbols, accepted, IJNT.

[5] F.J. Dyson, Some guesses in the theory of partitions, Eureka (Cambridge), 8:10-15, 1944.

[6] G. Gasper and M. Rahman, Basic Hypergeometric Series (Encyclopedia of mathematics and its applications; vol. 96), Cambridge University Press, 2nd ed., 2004.

[7] R.L. Graham, D.E. Knuth and O. Patashnik, Concrete Mathematics, A Foundation for Computer Science, Addison-Wesley, 2nd ed., 1998.

[8] K.Q. Ji, The combinatorics of k-marked Durfee symbols, Trans. AMS, to appear.

[9] S. Ramanujan and L.J. Rogers, Proof of Certain Identities in Combinatory Analysis, Proc. Cambridge Phil. Soc., 19:211-216, 1919.

[10] J. Riordan, Combinatorial Identities, John Wiley \& Sons, United States, 1968. 